\title{
Individualized Spatial Network Predictions Using Siamese Convolutional Neural Networks: A Resting-State fMRI Study of over 11,000 Unaffected Individuals
} Yuhui $\mathrm{Du}^{2}$, Zening $\mathrm{Fu}^{2}$, Thomas DeRamus², Eswar Damaraju², Bradley Baker²,3, and Vince D.

${ }^{2}$ Tri-Institutional Center for Translational Research in Neuroimaging and Data Science (TReNDS), Georgia State University, Georgia Institute of Technology, Emory University, Atlanta, GA, USA

${ }^{3}$ School of Electrical \& Computer Engineering, Georgia Institute of Technology, Atlanta, GA, USA

16 activity, given the inter-subject variability in brain anatomy, structure-function relationships, or life

17 experience. Many neuroimaging studies have demonstrated the potential of functional network connectivity

18 patterns estimated from resting functional magnetic resonance imaging (fMRI) to discriminate groups and

19 predict information about individual subjects. However, the predictive signal present in the spatial

20 heterogeneity of brain connectivity networks is yet to be extensively studied. In this study, we investigate,

21 for the first time, the use of pairwise-relationships between resting-state independent spatial maps to

22 characterize individuals. To do this, we develop a deep Siamese framework comprising three-dimensional

23 convolution neural networks for contrastive learning based on individual-level spatial maps estimated via

24 a fully automated fMRI independent component analysis approach. The proposed framework evaluates

25 whether pairs of spatial networks (e.g., visual network and auditory network) are capable of subject 26 identification and assesses the spatial variability in different network pairs' predictive power in an extensive 
27 whole-brain analysis. Our analysis on nearly 12,000 unaffected individuals from the UK Biobank study

28 demonstrates that the proposed approach can discriminate subjects with an accuracy of up to $88 \%$ for a

29 single network pair on the test set (best model, after several runs), and $82 \%$ average accuracy at the

30 subcortical domain level, notably the highest average domain level accuracy attained. Further investigation

31 of our network's learned features revealed a higher spatial variability in predictive accuracy among younger

32 brains and significantly higher discriminative power among males. In sum, the relationship among spatial

33 networks appears to be both informative and discriminative of individuals and should be studied further as

34 putative brain-based biomarkers.

35

36 Keywords-Resting-State fMRI, Siamese Neural Networks, Group-ICA Spatial Maps, Pairwise-Network

37 Interaction 


\section{Introduction}

Studies have investigated variability in human brain structure via standard assessment measures such

40 as cortical thickness, sulcal depth, and cortical folding across individuals [1,2,3,4]. Later studies discovered

41 the existence of relatively unique patterns in the human brain's functional organization. In particular,

42 significant variability in functional connectivity patterns (i.e., temporal dependence among brain network

43 timecourses) between groups of subjects (e.g., controls and patients) has been reported in the past decade

$44[5,6,7]$. Other studies have shown that functional connectivity patterns can be used to predict individual

45 traits [8]. Despite these findings, it is still unclear whether spatial relationships among functional networks

46 in the brain can be linked to individuals uniquely. Motivated by that, we investigate the spatial maps of

47 functional networks in this study and evaluate if the relationship between any two network spatial maps can

48 predict whether or not the maps come from the same subject.

49 Brain neural activity can be indirectly recorded by functional magnetic resonance imaging (fMRI)

50 based on the ensuing fluctuations in blood oxygenation level dependent (BOLD) signals [9,10]. Whole-

51 brain resting-state fMRI (rs-fMRI) measures BOLD fluctuations while an individual is at rest, i.e., subjects

52 are not performing an explicit task [11]. There has been significant interest in resting-state fMRI due to its

53 lower design complexity and ease of acquisition (relative to task fMRI, where an individual needs to be

54 able to perform a certain task) [12]. This advantage of rs-fMRI imaging renders it a widely used technology

55 leading to many studies, including those of patients with particular conditions (e.g., comatose individuals

56 or Alzheimer's patients) [13]. Multiple studies have also shown rs-fMRI data can be used to estimate

57 subject-level differences [14]. Various model- and data-driven methods (such as seed-based correlation

58 analysis [15], independent component analysis (ICA) [16,17], graph methods [18], and clustering

59 algorithms [19]) analyze whole-brain rs-fMRI scans to identify spatially distinct but functionally correlated

60 regions, also called resting-state networks (RSNs). Compared to other methods, ICA can identify

61 maximally statistically independent RSNs with less prior information. Also, it is able to capture artifacts 
62 and noise while separating these from the RSNs [9]. These advantages have led to the widespread use of

63 ICA to analyze rs-fMRI data.

64 Studies that utilize ICA for processing rs-fMRI data often analyze temporal patterns of brain activity,

65 such as the pairwise correlation between RSN-specific timecourses [20,21,22,7]. Despite the predominance

66 of studies on temporal characteristics of rs-fMRI data, the spatial characteristics of functional networks also

67 carry remarkable information and possess distinctive patterns for characterizing subjects [23,24,25]. Recent

68 studies have reported that ICA patterns can be used to classify group membership (e.g., patients versus

69 controls) [26]. In this article, we shed more light on a newer and yet more challenging problem, namely,

70 the discrimination of subjects solely from their underlying spatial map networks by learning inter-network

71 relationships in a verifiable way. To the best of our knowledge, this problem has not been studied before,

72 yet it has important implications for future studies of brain-based biomarkers and distributed networks.

73 The majority of studies that analyze resting-state connectivity rely on comparing functional patterns

74 between groups of people in some way. Some of these studies have tried to distinguish unaffected control

75 subjects from symptomatic ones, such as those with Alzheimer's disease [7,27], schizophrenia [8,28,29],

76 language-impairment [5], and autism [30,31]. In comparison, others have provided models to classify

77 subjects according to their sex [32,33] or age [34] using functional connectivity. Altogether, although these

78 studies attain individual-level predictions, they are ultimately focused on the use of group-level

79 (dis)similarity. However, as mentioned above, there has been much less focus on individual-level spatial

80 heterogeneity in brain functional interactions.

81 A few studies have shifted the focus to link such patterns to individuals $[6,14,35,8]$. More specifically,

82 Finn and colleagues [6] identify subjects based on their whole-brain and network-based functional

83 connectivity. They scanned the brain of 126 subjects over six sessions when performing working memory,

84 motor, language, and emotion tasks as well as at resting state. Then, for each subject, the functional

85 connectivity derived from each session was compared (using Pearson correlation) to the set of functional

86 connectivity of all subjects from other sessions. Another study [14] computed the variability in the intrinsic

87 functional connectivity of a small rs-fMRI dataset of 23 healthy subjects collected over six months with 
88 five scan sessions per individual. The individual variability in some brain regions was evaluated, including

89 frontal, temporal, and parietal lobes, and shown to be higher than in other regions. In a quite different

90 approach, a linear model was proposed in [35] for the prediction of a region of interest (ROI) time series

91 from another ROI time series. A model was fit to assess if the prediction is unique for each subject, i.e., the

92 prediction pattern was distinctive between 27 individuals. Such models suffer from a few fundamental

93 shortcomings. First, they usually require multiple scans from each subject for proper training, which is

94 costly and time-consuming; for instance, it took over six months for the authors in [14] to complete the data

95 acquisition phase. Second, due to the lack of high-sample-sized datasets for such longitudinal models, it is

96 hard, if not impossible, to train the high-capacity models that are typically better at capturing complex

97 features and, thus, such models are not otherwise generalizable due to overfitting [36]. Furthermore, all

98 these studies disregard the spatial maps of functional brain networks in their analysis, while several studies

99 have shown such information can be used as biomarkers to characterize individuals $[37,38,39]$.

100 Here we use a large resting-state fMRI dataset to identify discriminative features of brain activity

101 between unaffected individuals to address the shortcomings above. Our goal is to characterize individuals

102 based on high-level features learned from their functional brain network spatial patterns and from pair-wise

103 comparisons between subjects given a pre-selected network pair. We are also interested in observing which

104 specific networks are more informative for such task. Accordingly, we develop a deep neural network-

105 based framework that can detect subjects based on high-level difference features in the spatial patterns of

106 their functional networks, when those networks are different (e.g., auditory and visual networks) for each

107 subject. Our assumption is that there exists some high-level spatial pattern that underlies all such functional

108 networks but is unique to each person. Thus, we hypothesize that subjects can be differentiated from each

109 other by contrasting such a pattern if it can be extracted and represented in a latent feature space where the

110 distances make biological sense. In other words, our aim is to learn a functional brain pattern that 1) is

111 unique to the individual brain and 2) can be mapped to a unified feature space where differences in subject

112 labels translate, by design, to L1 distances. In that respect, it does not matter which network of the brain we

113 are selecting from an individual as long as we can infer the latent feature(s) from it, which can then be used 
114 to capture differences. Given the possibly nuanced complexity and unpredictable nature of such spatial

115 (dis)similarity features, it is fair to conjecture they may be successfully learned via deep neural network

116 model representations.

117 Our approach of characterizing brain samples using a pair of functional brain networks is different from

118 the few previous studies in several ways. First, we develop a framework to capture complex functional

119 patterns in the brain and do so in a region agnostic manner (i.e., regardless of the selected brain network

120 pair). Second, we transform the problem of subject identification into subject comparison by taking

121 advantage of the Siamese architecture [40,41], thus reducing the original multiclass problem into a binary

122 classification problem (class 1 (or positive) if two input spatial maps are from the same subject, class 2 (or

123 negative) otherwise), which is moderately easier to train. Third, we leverage the nature of this task to obtain

124 a relatively large augmented dataset of pairs of subjects, effectively boosting the predictive power of our

125 end-to-end trained models. Furthermore, our models do not require multiple scans per subject, which is a

126 limitation in some previous studies. Finally, as will be discussed, each trained model works on a preselected

127 pair of functional networks and, thus, can capture relationships between brain regions, contrary to previous

128 work that is limited in this regard.

129 This paper is organized as follows. In section 2, we describe the data and the procedure underlying data

130 collection and preprocessing and subsequently introduce our model in more detail. In section 3, we evaluate

131 our model on a held-out test set using the Monte Carlo cross-validation approach and shed more light on

132 age and sex differences in its performance. Section 4 reflects on our observations and the model's

133 performance. Finally, section 5 concludes our paper and suggests future directions to continue this line of

134 research.

\section{2. Materials \& Methods}

136 2.1. Participants

137 We retrieved the resting-state fMRI dataset from the UK Biobank [42]. At the time of retrieval, this 138 dataset included 19831 subjects, out of which 13668 were self-reported as healthy (unaffected) adult 
139 participants. The subject fMRI scans underwent quality control, and subjects were excluded if the scans

140 met the following criteria: marked as unusable by UK Biobank, visual inspection of mean maps for gross

141 anomalies, absolute framewise displacement (FD) higher than $0.3 \mathrm{~mm}$, Matthews correlation coefficient

142 (MCC) between the binarized study-specific mask and the subject mask lower than 0.8, and failure to

143 complete ICA estimation. 11754 subjects were finally retained for the analysis after quality control, with

144 the included participants' ages ranging from 45 to $80(62.56 \pm 7.38)$ years. The dataset was well balanced

145 in terms of the participants' sex (c.f. Table 1).

Table 1. Subjects' demographics.

\begin{tabular}{|c|c|c|c|c|c|c|c|c|}
\hline & Population & \multicolumn{8}{|c|}{ Age (years) } \\
\cline { 3 - 9 } & Number & Mean & SD & Min. & $\mathbf{2 5 \%}$ & $\mathbf{5 0 \%}$ & $\mathbf{7 5 \%}$ & Max. \\
\hline All & $11754(100 \%)$ & 62.56 & 7.38 & 45 & 57 & 63 & 68 & 80 \\
\hline Male & $5772(49 \%)$ & 63.08 & 7.54 & 45 & 57 & 64 & 69 & 80 \\
\hline Female & $5982(51 \%)$ & 62.07 & 7.19 & 46 & 56 & 62 & 68 & 80 \\
\hline
\end{tabular}

\subsection{Data Acquisition \& Preprocessing}

All participants were scanned once by a 3-Tesla (3T) Siemens Skyra scanner with a 32-channel receive

head coil, all acquired in one site. A gradient-echo echo planar imaging (GE-EPI) paradigm was used to

151 factor of 8 (i.e., eight slices were acquired simultaneously), no iPAT, fat saturation, flip angle (FA) $=52^{\circ}$,

152 spatial resolution $=2.4 \times 2.4 \times 2.4 \mathrm{~mm}$, field - of - view $(\mathrm{FOV})=(88 \times 88 \times 64$ matrix $)$, repeat time $(\mathrm{TR})$

$153=0.735 \mathrm{~s}$, echo time $(\mathrm{TE})=39 \mathrm{~ms}$, and 490 volumes. Subjects were instructed to stare at a crosshair

154 passively and remain relaxed, not thinking about anything, during the six-minute and ten-second resting-

155 state scanning period.

156 The preprocessing steps performed by UK Biobank are as follows. An intra-modal motion correction

157 tool, MCFLIRT [43], was applied to minimize the distortions due to head motion. Grand-mean intensity

158 normalization was used to scale the entire 4D dataset by a single multiplicative factor to compare brain

159 scans between subjects. The data were filtered by a high-pass temporal filter (Gaussian-weighted least-

160 squares straight-line fitting, with $\sigma=50.0 \mathrm{~s}$ ) to remove residual temporal drifts. Geometric distortions of 
161 EPI scans were corrected by using the FSL's Topup tool [44]. EPI unwarping is followed by a gradient

162 distortion correction (GDC) unwarping phase. Finally, structured artefacts are removed by ICA+FIX

163 processing (Independent Component Analysis followed by FMRIB’s ICA-based X-noiseifier [45,46,47],

164 with no lowpass temporal or spatial filtering up to this point. More details on the UK Biobank imaging

165 protocol and preprocessing steps can be found in [42]. In addition, the data were then normalized to an MNI

166 EPI template using FLIRT followed by SPM12, old normalization module. Finally, the data were smoothed

167 using a Gaussian kernel with FWHM = 6mm.

168 2.3. Group Independent Component Analysis

169 We applied fully automated spatially constrained ICA using the NeuroMark approach [48] on the 4D

170 preprocessed UK Biobank rs-fMRI data from Section 2.2. In the Neuromark approach, a template of

171 replicable independent components (ICs) was constructed after spatially matching correlated group-level

172 ICs between two healthy control fMRI datasets - genomics superstruct project (GSP) and human

173 connectome project (HCP). The estimated network template was then used as a prior for a spatially

174 constrained ICA algorithm applied to each UK Biobank subject individually. This identified 53 functionally

175 relevant resting-state networks (RSNs) for each individual that are maximally spatially independent (see

176 Fig. S1 and Table S1). Each subject-specific RSN is represented by a spatial map of size $53 \times 63 \times 52$ voxels

177 and its associated time course of 490-time points. RSNs are grouped into seven domains, namely subcortical

178 (SC), auditory (AU), sensory-motor (SM), visual (VI), cognitive control (CC), default mode (DM), and

179 cerebellar (CB), by functional similarity (see Fig. 1). This work used the subject-specific spatial maps

180 (henceforth referred to as networks) as input to our model and a basis for all subsequent analyses.

$181 \quad$ 2.4. Generating Input Pairs

182 We split our preprocessed data by subjects into training, validation, and test sets with a proportion of $18360 \%, 20 \%$, and 20\%, respectively (see Table S2-4 for the statistics of subjects in each set). The number of 184 same-subject pairs created for each set was equal to the total number of subjects in each set, and all these 185 pairs were labeled 'class 1.' Likewise, an equal number of different-subjects pairs (labeled 'class 2') were 
186 created for each set by randomly selecting subjects from the corresponding set (for a total of $N$ pairs out of

$187\left(\begin{array}{l}N \\ 2\end{array}\right)$ possible pairs, where $N$ is the total number of subjects in each set). As discussed before, input pairs are

188 comprised of two networks instead of one. This is important as, otherwise, instead of learning patterns that

189 govern the relationships within networks, the model gradually (i.e., within the course of training) tends to

190 learn to make an elementwise comparison of the input voxels. We trained one model for each pair of the

19153 functional networks using the TReNDS high-performance computing GPU cluster. In total, $\left(\begin{array}{c}53 \\ 2\end{array}\right)=1378$

192 models were trained.

\section{2.5. Model Architecture}

194 We provide an end-to-end trainable deep learning model for learning a non-linear dissimilarity metric 195 based on patterns of spatial relationships in functional networks that characterize each subject. We use 196 balanced (i.e., same-subject vs. different-subject) preprocessed input-pairs for training, and the objective is

197 to adjust network weights such that input pairs that belong to the same subject produce a low dis-similarity

198 score whereas those pertaining to different subjects generate a large score. Our proposed framework 199 comprises two major building blocks, one based on the convolutional neural networks (CNNs), which are 200 superior in capturing high-level features from imaging data modalities [49], and the other based on a 201 Siamese network that can efficiently generate features for differentiating inputs [40,41]. In the following 202 sub-sections, we shed more light on the architecture of each module. 

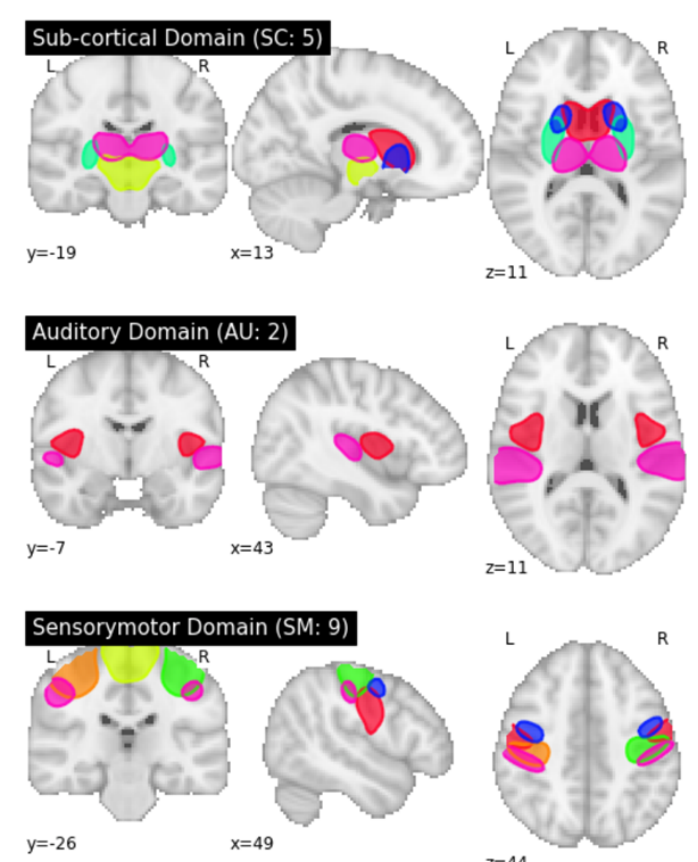

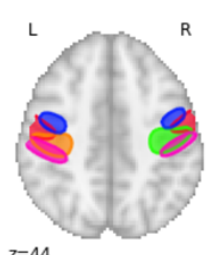

$z=44$
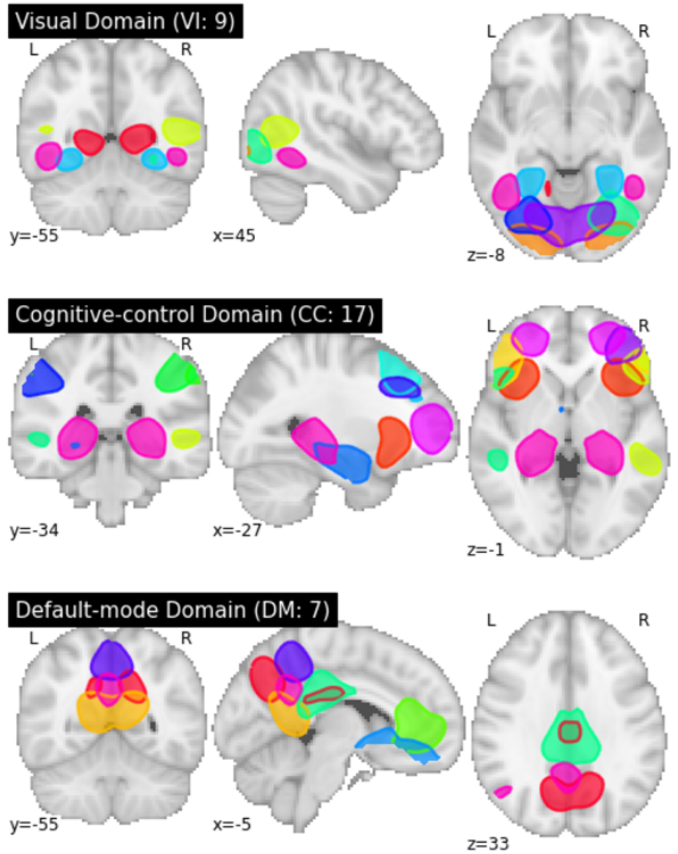



Fig. 1. Group ICA-derived spatial maps. Spatial maps are grouped into seven domains, subcortical (SC), auditory (AU), sensory-motor (SM), visual (VI), cognitive control (CC), default mode (DM), and cerebellar (CB), each of which contains $5,2,9,9,17,7$, and 4 networks, respectively.

\subsection{Convolutional Neural Networks (CNNs)}

2D CNNs and, more recently, 3D CNNs have gained attention in various domains that involve image

210 these features should be comparable between different subjects, even if they are derived from different

211 spatial maps. This is indeed a crucial component of our design since using spatial maps from the same

212 networks drives the training course towards a point in the parameter space that corresponds to a voxel-to- 
213 voxel verbatim comparison. In other words, instead of learning a pattern that characterizes brain activities,

214 the model will tend to see if the two images are identical voxel-wise. We therefore prevent this by utilizing

215 network spatial maps as input and two CNN-based child networks that can generate informative features

216 linked to each other via a Siamese architecture, as will be discussed shortly.

217 Fig. 2 depicts the architecture of the employed 3D ConvNet that extracts features from a supplied spatial 218 map with a resolution of $53 \times 63 \times 52$ voxels. We used three convolutional layers with 16 , 32, and 64 filters, 219 respectively, and used kernels of $3 \times 3 \times 3$ for each of the layers. We used ReLU activations for both 220 convolutional and later fully connected layers. Furthermore, we augmented the convolutional layers with 221 max-pooling layers (with a kernel size of $2 \times 2 \times 2$ and stride of 2 ) to reduce the feature maps dimensionalities.

222 The features in the last feature map are flattened and then fed into a single fully-connected layer with a 223 ReLU of size 128 neurons. We used 3D and 1D batch-normalization as a regularization for all layers. This 224 is especially important due to the large dimensionality and complexity of the model. The DL encodings 225 (i.e., final feature values) serve as a summary of the input 3D spatial map, capturing the non-linear pattern 226 of functional relationships underlying the input brain network pair when trained based on a loss that 227 quantitatively factors in our goal, as discussed in the next section.

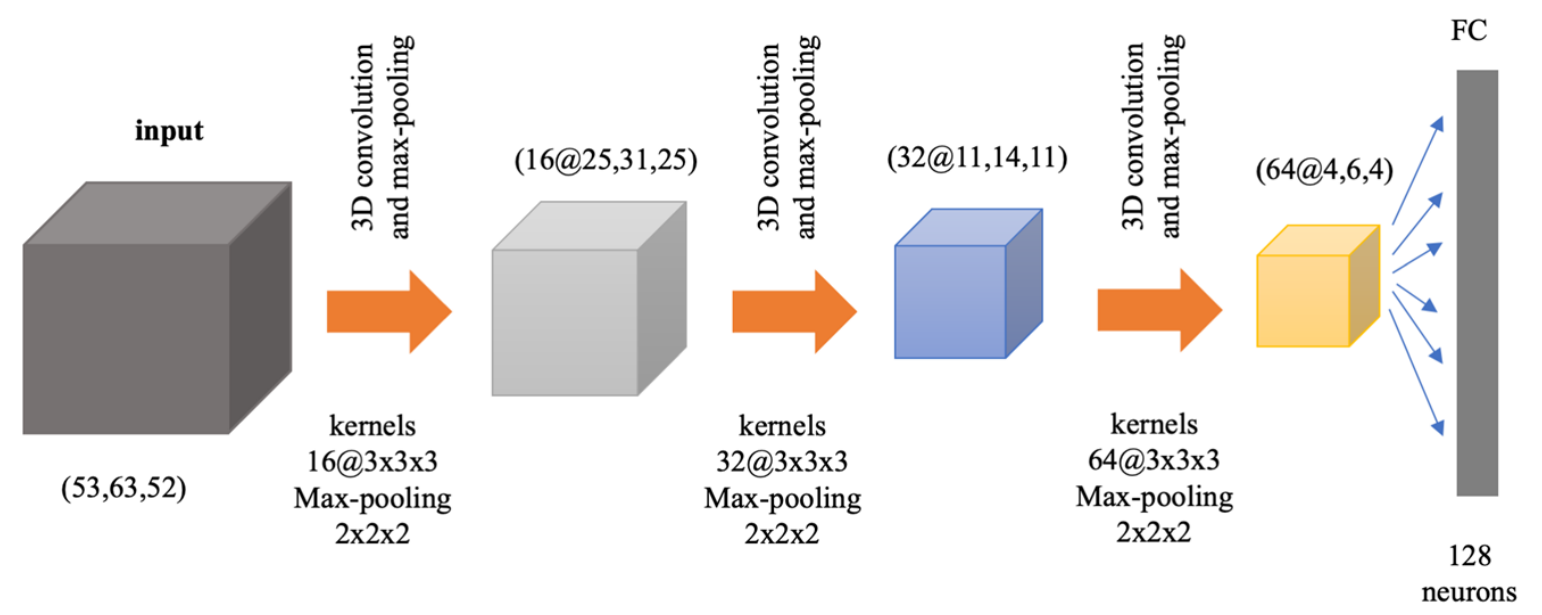

Fig. 2. CNNs architecture. The CNNs block consists of three $3 \mathrm{D}$ convolutional layers, with kernels of sizes $3 \times 3 \times 3$, each of which is followed by a max-pooling layer with a kernel of size $2 \times 2 \times 2$. 


\subsection{Siamese Architecture}

231 For our brain-network classification task, we use a modified Siamese-based CNN model, seeking to 232 map input image pairs to a shared target space in which both spatial map images are comparable. In other

233 words, we learn separate mappings that transform the (spatial) input spaces of two different functional brain

234 network maps into a shared high-level space. In that space, the distance between subjects (irrespective of 235 the brain network region) represents how closely related their corresponding functional networks are. By 236 training specifically on different brain network pairs, we ensure that closely related network representations

237 learned by the model are not merely driven by spatial similarity but, rather, by having their origin on the 238 same subject. Fig. 3 shows the block diagram of our proposed model. It is comprised of two CNN-based 239 child networks with identical architectures (referred to as sub-networks hereon), but not sharing weights.

240 Each sub-network takes a different functional brain map network image, namely $X_{1}$ and $X_{2}$ (e.g., visual 241 network and auditory network), to generate consistent and comparable representations $R_{1}\left(X_{2}\right)$ and $R_{2}\left(X_{2}\right)$, 242 respectively. Then, optimizing for a Siamese-based architecture using the binary cross-entropy loss drives 243 parameters of the $\mathrm{CNN}$ sub-networks towards a point in the parameter search space where projections lie 244 in distant locations when subjects are different. Our experimentation suggests that this is more easily 245 attained with independent, rather than tied, weights for each child network. This choice is also driven by 246 the problem at hand. Using shared weights is a common approach if the objective is to learn a generic 247 representation for both input data modes (here, brain network spatial maps), whereas using independent 248 weights is more suitable if learning mode-specific representations instead. Thus, the use of separate weights 249 is more appropriate for our objective of training models that learn if two modes (i.e., brain network spatial 250 maps) correspond to the same subject or not. Additionally, we assume that a softmax-normalized L1

251 distance, trained through the binary cross-entropy loss, will induce both sub-networks to converge into a 252 shared feature space.

253 We train this network for 200 epochs using stochastic gradient descent with a binary cross-entropy loss 254 and a learning rate of 0.001 . The model is given by $\operatorname{los} s=-t \log (S)-(1-t) \log (1-S)$ where $t$ is the 
255 true label, $S=\operatorname{sigmoid}\left(\sum w_{i} d_{i}\right), d_{i}=\left|R_{1}\left(X_{2 i}\right)-R_{2}\left(X_{1 i}\right)\right|$, and $i$ is the dimensionality index of the feature

256 space. The training is stopped using the early-stopping strategy of no improvement in validation accuracy

257 after 14 consecutive epochs. Furthermore, we repeat the training course three times and select the best

258 validation model (i.e., the one with the highest validation accuracy) for testing on the held-out test data.

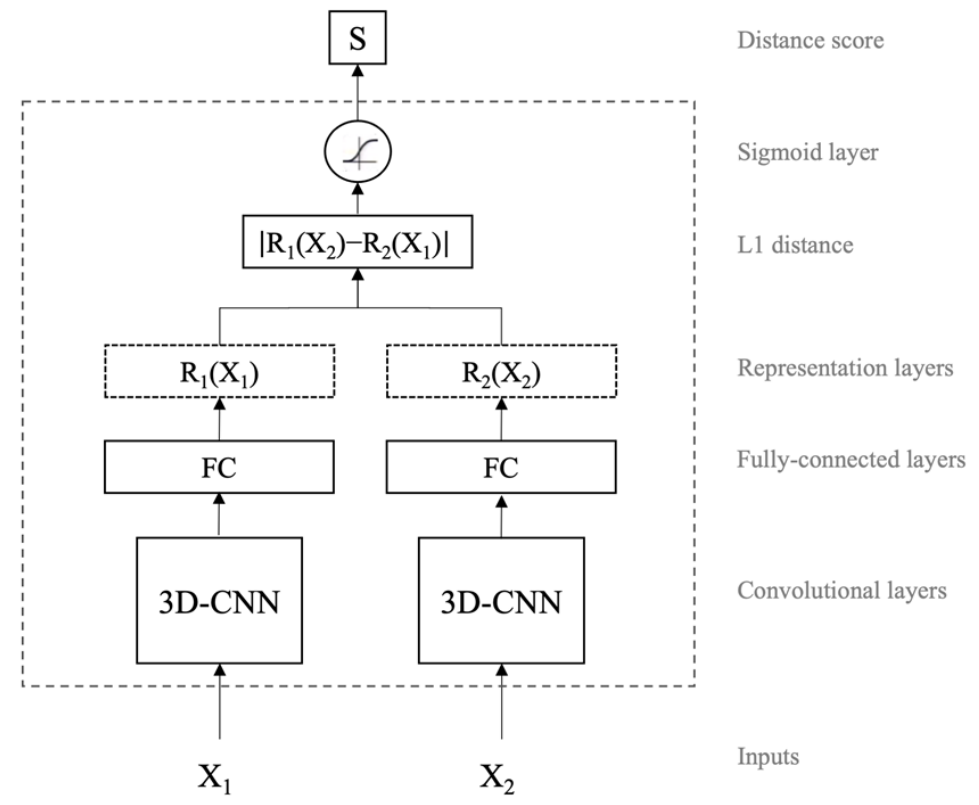

Fig. 3. High-level architecture of the proposed model. Siamese networks take two images, $X_{1}$ and $X_{2}$, and learn a distance function between the two vector representations of the input images. The model outputs distance score close to one if the two inputs are from two different objects (in this case, different subjects) and close to zero otherwise. The weights of the CNN child networks are updated independently and are not tied/shared.

\section{Experiments}

In this section, we evaluate the performance of the proposed model when trained on different pairs of

263 functional brain networks, on unseen (held-out) test datasets. In section 3.1, we compare and contrast the 264 accuracy achieved when we select networks from different domains. In addition, we discuss and analyze 265 the prediction performances across three held-out test set sample cohorts according to the class labels we attempt to predict: network pairs from the same subjects, network pairs from different subjects, and the 267 entire (aggregate) set of all network pairs (same and different subject pair samples together). Using these 
268 cohorts enables us to evaluate our model architecture's performance under different scenarios and provides

269 insight into brain function. In sections 3.2 and 3.3, we analyze the relationship between cognitive features,

270 age and sex, and model performance.

271 3.1. Analysis of Performance in Different Functional Network Domains

272 To investigate potential variation in model performance by network pairs, we evaluated the proposed 273 model on the entire held-out test set (i.e., the cohort containing all subject pair samples), which is comprised 274 of an equal number of same-subject and different-subject network-pair samples. Fig. 4A shows a heatmap 275 of the mean model accuracies between domains (see the fine-grained brain network connection-level 276 accuracies in Fig. S2). According to the heatmap, there exist spatial network relationship patterns with 277 strong prediction capability stemming from SC-SC, SC-CC, SC-DM, SC-VI, VI-VI, and DM-DM domain278 pairs. On the other hand, network pairs including an auditory network appear to contain less discriminative 279 (yet still significant) features. Overall, it turns out that the amount of discriminative information that can be 280 captured from a given network pair is at least partly domain-dependent (see Table S5 for a two-sided two281 sample $t$-test of the mean prediction accuracy between domains and the corresponding $p$-values). 282 Furthermore, to assess the reproducibility and generalizability of the results, we repeat our training-testing 283 approach using the Monte Carlo cross-validation (MCCV) approach with five repeats (the held-out test set 284 changes for each repeat). Fig 4B shows a boxplot of the corresponding accuracies for each pair of networks 285 that fall under SC-SC and AU-AU domain pairs (i.e., the highest and the lowest performant domain pairs 286 according to Fig. 4A, respectively). From the figure, it is evident that our results are reproducible up to a 287 negligible variation in model performance. 

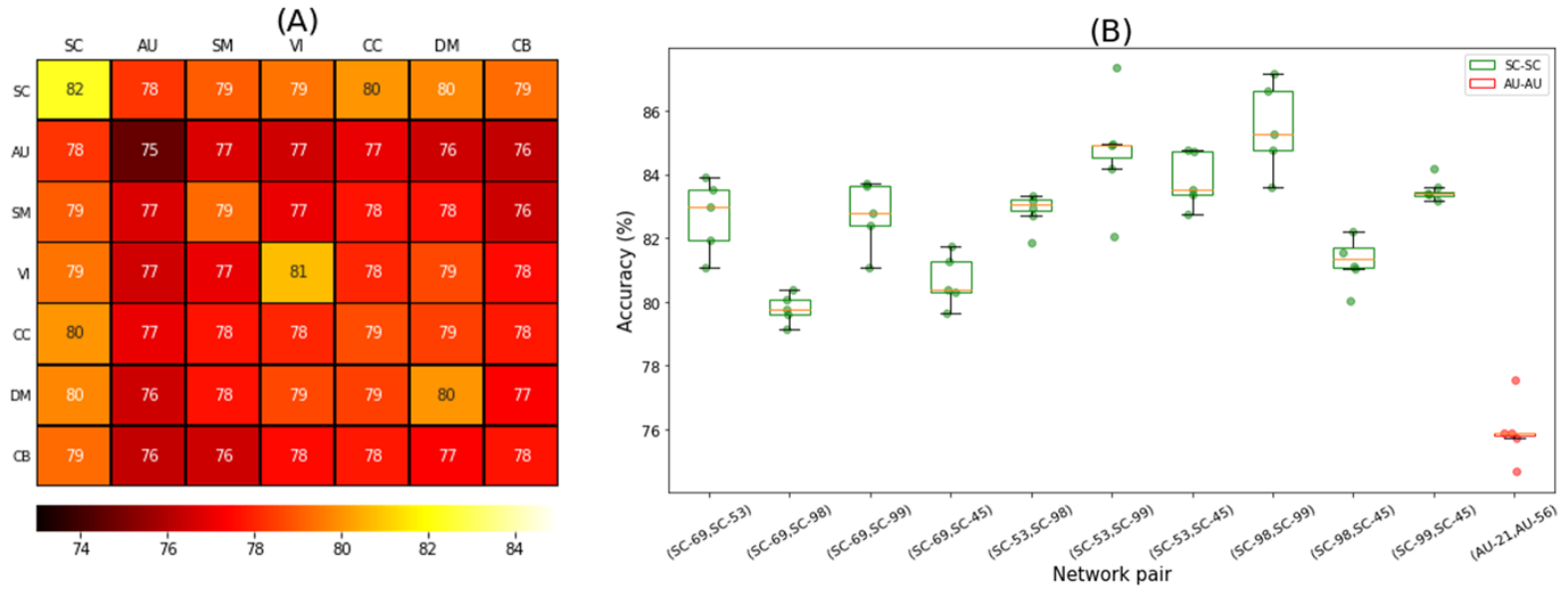

Fig. 4. (A) Mean domain accuracy heatmap. Each cell shows the mean accuracies of all models trained on spatial networks belonging to the indicated domain-domain pair. The resulting mean accuracies show that domains have different predictive power for characterization of subjects based on brain activity. A comparison of SC to the other domains reveals that its underlying networks yield the most discriminative features. On the other hand, the AU domain appears to produce the least discriminative features relative to other regions in the brain. (B) Boxplot for the accuracies derived from the MCCV evaluation. The results show low variation across the five repeats for pairs of networks that fall under SC-SC and AU-AU domain pairs, which provides supporting evidence for the generalizability of the proposed model. Green and red boxes represent SC and AU network pairs, respectively. The dots are the results of MCCV for each network pair. The X-axis indicates the Neuromark network ids.

We were also interested in investigating whether any network pairs were more feature-rich for either of the same- vs. different-subject cohorts in the classification task. This is an important question as significant differences can serve to support recommendation guidelines for optimally choosing certain network pairs for different goals. In light of that, we experimented by partitioning the test samples into a

294 cohort of network pairs from the same subjects and a cohort of pairs with different subjects. Figures 5A and

295 5B depict the spatial connectograms of the top $2 \%$ network pairs with the highest sensitivity (we treat same-

296 subject samples as the positive class) and specificity, respectively (see Fig. S3A and S3B for the full results).

297 We grouped connectograms based on their functional domains, i.e., SC, AU, SM, VI, CC, DM, and CB, 298 which contain $n=5,2,9,9,17,7$, and 4 networks, respectively. Specifically, Fig. 5A shows that the highest 299 sensitivities are mostly from brain regions in the subcortical and cognitive control domains. While 300 according to Fig. 5B, brain regions in the visual domain exhibit the highest specificities. Thus, comparing 
301 the two connectograms suggests that different domains offer distinct patterns of contribution towards 302 identifying same- vs. different-subject pairs.
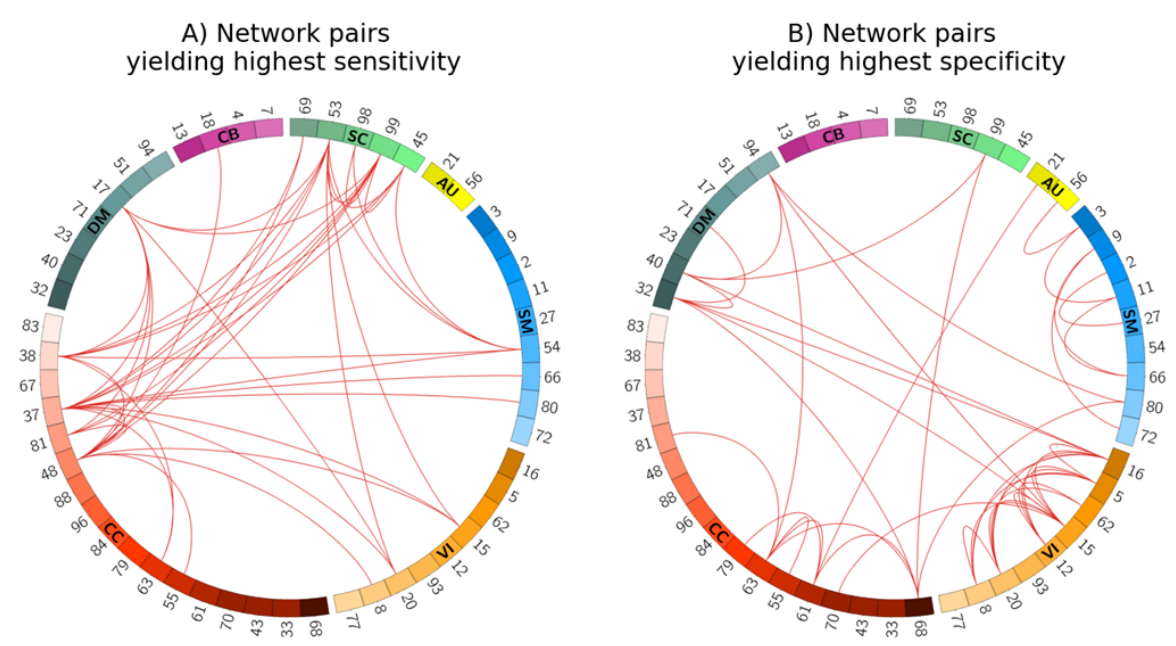

Fig. 5. Spatial connectogram of sensitivity (A) and specificity (B) highlighting the top $2 \%$ network pairs. Sensitivity and specificity show the percentage of network pair samples correctly identified as the same- or different-subject cohorts, respectively. Fig. 5A suggests that networks from the subcortical (green) and cognitive control (red) domains are among the most useful for characterizing same-subject samples, both intra- and interdomain. On the other hand, the high specificity of VI-VI network pairs (orange) in Fig. 5B indicates better characterization of different-subject samples. The numbers shown on the connectograms represent the specific Neuromark RSN network indices (See Table S1 for a list of the network names). The connectograms were generated using the Circos tool [50].

\subsection{Impact of Sex on Performance}

We also evaluated the role of sex on the prediction performance separately for the same- and differentsubject cohorts. First, we considered the same-subject cohort and compared male vs. female subcohorts within it. We observed that the model's sensitivity when two networks are selected from male subjects is larger than when they are selected from female subjects, which was the case for $71 \%$ of network pairs (See Fig. S4). This suggests that male functional networks have more uniquely identifying individual patterns,

311 especially in the cerebellar domain, compared to females. This finding is consistent with studies of brain 312 structure that maintain males' brains have more variability than females [51,52]. Indeed, higher variability 
313 in brain structure is conducive to the existence of more distinct patterns in males that help characterize male

314 subjects' identities more accurately.

315 To better visualize the difference in performance, we subtracted the female cohort's sensitivity scores

316 from that of males for each network pair and selected the network pairs corresponding to the highest

317 (positive) and lowest (negative) 1\% score difference, as illustrated in Fig. 6A. According to the figure, from

318 the pairs in which males have higher sensitivity (i.e., the red arcs), the superior parietal lobule (SPL)

319 network (indicated as SM27) shows more outgoing arcs in the connectogram. This may suggest that the

320 SPL network activity varies more (or, is more uniquely identified) in males than females. To the best of our

321 knowledge, this is the first time such a pattern has been identified. Studies have shown that the SPL network

322 is linked to spatial processing tasks, especially in mental rotation [53,54]. Another interesting research has

323 reported stronger activity of these networks among males [55]. Altogether, these observations seem to

324 generally indicate that while men might do better at spatial orientation tasks than women, there is more

325 variation to their brain functional engagement in this task than women. Hence, the significance of SM27 as

326 a functional brain activity biomarker for classification of males from females should be considered.

327 Likewise, among networks with the lowest (most negative) sensitivity difference between males and

328 females_(i.e., the blue arcs in Fig. 6A), CC70 presents the most outgoing arcs, suggesting this network is

329 more discriminative in females.

330 Finally, we considered the set of different-subject samples. This set naturally splits into same-sex (male-

331 male or female-female pairings) and different-sex (male-female pairings) subcohorts, for which we

332 conducted assessments analog to those shown in the male vs. female discussion above. Interestingly, we

333 observed higher specificity in the different-sex cohort for $86 \%$ of network pairs. According to the results

334 of this assessment, the model performs better (higher specificity) when samples belong to different sexes,

335 for most network pairs. This is likely because our model is capable of learning sex-rich features from the

336 network spatial map patterns, even though sex has not been used as an input to our model. The

337 corresponding highest and lowest $1 \%$ specificity differences are visualized in Fig. 6B. For the most negative

338 differences (same-sex specificity lower than for different-sex), we observed that network pairs including 
339 either the default mode (especially DM32 and DM51) or the cognitive control networks had the most

340 outgoing links, suggesting more unique variations in the different-sex cohort. For those network pairs with

341 positive specificity differences, on the other hand, the CC networks, especially CC84, appear more

342 frequently in the connectogram, suggesting that more unique patterns occur in the same-sex cohort when

343 network pairs include a CC network.
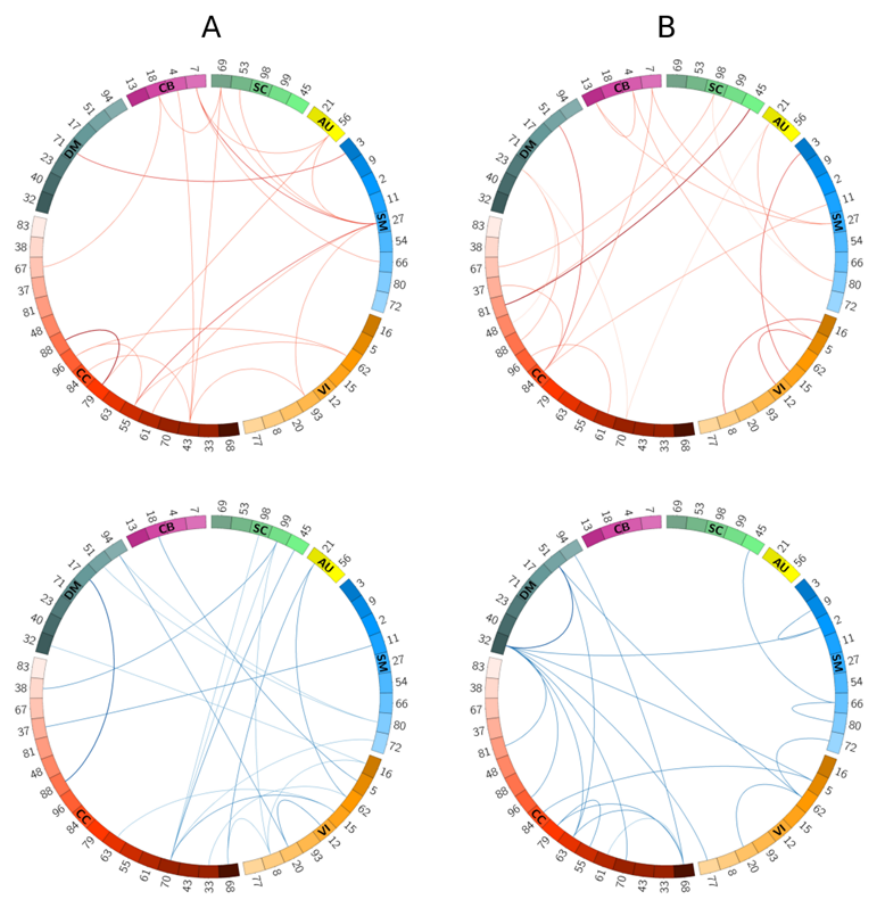

Fig. 6. Spatial connectogram of difference in sensitivity $(A)$ as well as the difference in specificity $(B)$ between sex-based groups highlighting the top $1 \%$ positive (top row) and negative (bottom row) differences. Fig. 6A: scores are calculated by subtracting sensitivities of the female subcohort from the male subcohort. SM27, when paired with other domains, produces higher specificity in males, while CC70 yields higher specificity in females. Fig. 6B: scores are calculated by subtracting specificities of different-sex from same-sex subcohorts. The SM and $\mathrm{CB}$ networks produce higher sensitivities for different-sex samples, whereas the $\mathrm{CC}$ networks predict better in the same-sex subcohort. Blue and red color lines represent the two ends of the difference spectrum. Numbers represent indices for the ICA-derived Neuromark RSNs. The connectograms were generated using the Circos tool [50].

Furthermore, we assessed the previous experiments' results from a statistical perspective (see Table

347 S6). We performed a two-sided two-sample $t$-test which shows the significance of the mean sensitivity or 348 specificity difference between the two sub-cohorts of each sex-based assessment. Accordingly, we observed 
that the mean sensitivity in the male sub-cohort is significantly different in the female sub-cohort, and the mean specificity in the different-sex sub-cohort is significantly different from the same-sex sub-cohort.

351 These seem especially true for the cognitive control domain.

352 3.3. Impact of Age on Performance

353 Next, we analyzed the relationship between age and model performance. Similar to experiments in the 354 previous section, we divided the test set into same- and different-subject cohorts. We computed the 355 sensitivity of subjects of ages below 52 and above 72, which make cohorts of size 260 and 268 subjects, 356 respectively. Our results revealed that the younger cohort's prediction performance is significantly higher 357 than that of the older cohort for $66 \%$ of network pairs (see Fig. S5A). This is especially the case for the 358 cognitive control $(p<2 e-22$, see Table S7), sensory-motor $(p<6 e-18)$, and default mode $(p<1 e-11)$. In

359 a similar experiment, we computed the specificity for all pairs of networks within the different-subject 360 cohort for 139 and 158 subjects with age below 57 and above 69 years old, respectively (see Fig. S5B). For 361 most domains, the model performance for younger brains appear to be stronger than older ones, especially 362 for the cognitive control $(p<4 e-8)$ and cerebellar $(p<2 e-8)$ domains.

363 To shed more light on the role of age on our model performance, we computed pairwise sensitivity and 364 specificity differences between the young-old cohorts and then picked the highest and lowest $1 \%$ of the 365 resulting scores. Fig. 7A and 7B show these differences for the sensitivity and specificity metrics, 366 respectively. Based on these figures, from the pairs with the largest sensitivities (the arcs colored in red) 367 networks CC61, CC79, and SC69 when paired with a number of other networks appear to be more 368 discriminative. On the other hand, among the networks with the lowest sensitivity difference score (arcs 369 colored in blue) networks CB18 and SC98 are linked with many outgoing arcs suggesting that these 370 networks contain more unique patterns within the cohort of old subjects. Altogether, the aforementioned 371 networks can be used in age-related tasks where we are interested in comparing intra-subject networks.

372 Likewise, considering Fig. 6.B, networks from the CC and SM domains when linked with other networks 
373 (Fig 7B, bottom row) along with the (CC70, VI5) network pair (Fig 7B, top row) are well suited for age-

374 related tasks using patterns of networks between subjects (e.g., classifying younger vs. older individuals).
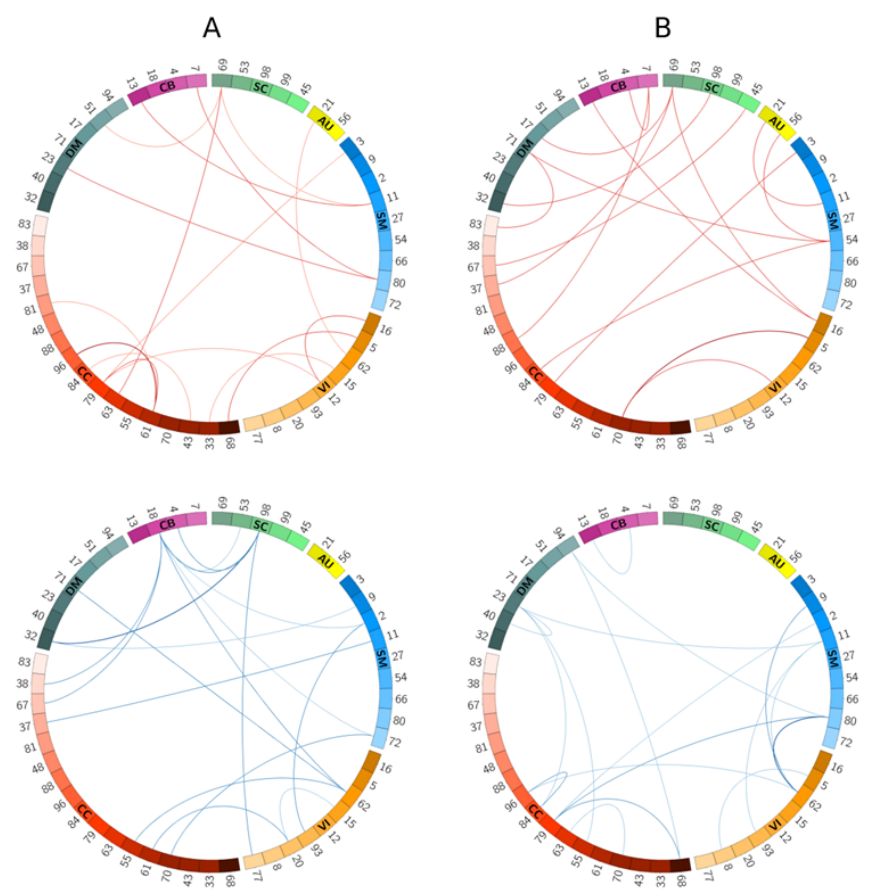

Fig. 7. Spatial connectogram of difference in sensitivity (A) as well as the difference in specificity (B) between young and old groups with the highest (top row) and lowest (bottom row) $1 \%$ values. All the connectograms show the difference scores computed from deducting old subjects from young subjects. Fig. 7A suggests CC61 and CC79, and SC69 networks contain more discriminative features in young subjects. On the other hand, networks of CB18 and SC98 perform better when subjects are old. Fig. 7B shows the network pair CC70-VI5 has the largest specificity difference between young and old subjects. In contrast, the combination of the CC and SM networks can better predict the dis-similarity metric when subjects are older. The connectograms were generated using the Circos tool [50].

\section{Conclusion}

In this study, we showed for the first time that pairwise-relationships between ICA-based spatial maps can predict whether or not two networks belong to the same subject. As such, we proposed a Siamese-based model that extracts individualized patterns by pairwise comparison of spatial network maps (e.g., an auditory and a visual network). In our model, we combined a Siamese architecture with convolutional neural networks to learn high-level features describing 3D functional brain network maps suitable for the downstream comparison of subjects in our prediction task. Using the features extracted from the CNN 
networks, our model generated a non-linear distance metric representing the chance the two networks are from same or different subjects. Our results of training the proposed model for all possible network pairs showed that different pairs of functional networks contributed differently to the network prediction task.

This was especially true when networks were selected from the subcortical domain (with the highest accuracy) or the auditory domain (with the lowest accuracy). The model's high performance in subcortical networks suggested the superiority of such networks being used for future end-point tasks, such as age classification, brain fingerprinting, etc., under the proposed framework.

We also provided guidelines for neuroimaging-based prediction tasks by investigating which network pairs were more feature-rich in each of the same-subject and different-subject cohorts. We observed that networks from the subcortical and cognitive control domains, and especially their combinations,

393 demonstrate more variability in the cohort of same-subject samples. Therefore, we suggest such pairs of

394 networks are well suited for analyzing spatial interactions, for example, for predicting diseases. On the

395 other hand, in the different-subject cohort, network pairs from the visual domain resulted in more accurate models and can later be used for a task that scrutinizes networks between subjects, e.g., for identifying

397 same-sex samples.

Further analysis of our results revealed that the performance of our model depends on subjects' age and sex. From the sex-based assessments, we observed in most cases (71\%) the model performed better in males corroborating the previous studies' findings, which showed that males' brain networks have larger

402 functional activity in the SPL network varies more within males than females. Future assessment and study 403 of potential sex bias in these findings is warranted, and approaches such as [56] could be easily adapted to 404 our framework for such purposes. Moreover, for $86 \%$ of network pairs, we observed higher specificity 405 among different-sex subjects than same-sex subjects. This suggests higher discriminative patterns between 406 networks of different sex compared to networks of the same sex. When it comes to the impact of age, our 407 results showed that the prediction performance among the younger cohort is significantly higher than that 408 of the older cohort for most network pairs. Overall, our age and sex-related findings showed that our model 
could learn sex and age-rich features from the spatial maps, even though sex and age have not been

explicitly used as an input to our model.

411 Overall, despite the widespread use of timecourse-derived information (i.e., functional connectivity) in

412 prediction-focused neuroimaging studies, spatial maps are feature-rich data sources that can serve as

413 surrogates to timecourse data or even be used as complementary source input. As future work, we encourage

414 to utilize the same approach to investigate whether similar patterns exist among diseased and healthy cases.

415 Moreover, we hypothesize that our model can serve as the first step towards brain fingerprinting through

416 discovering unique functional patterns that can characterize people in a unique way.

\section{Author Contributions}

418 Reihaneh Hassanzadeh proposed and implemented the main idea, trained models, analyzed the results,

419 and drafted the paper. Rogers F. Silva, Yuhui Du, Mustafa Salman, Anna Bonkhoff, Zening Fu, Thomas

420 DeRamus, Eswar Damaraju, Bradley Baker, and Anees Abrol preprocessed the data, performed quality

421 checks, and extracted the ICA components. Vince D Calhoun served as the PI and our guide throughout the

422 project. All authors provided input and edits to the manuscript.

\section{References}

1. Mangin JF, Rivière D, Cachia A, Duchesnay É, Cointepas Y, Papadopoulos-Orfanos D, et al. A framework to study the cortical folding patterns. Neuroimage. 2004; 23: S129-S138.

2. Bürgel U, Amunts K, Hoemke L, Mohlberg H, Gilsbach JM, Zilles K. White matter fiber tracts of the human brain: Three-dimensional mapping at microscopic resolution, topography and intersubject variability. Neuroimage. 2006; 29(4): 1092-1105.

3. Smith S, Duff E, Groves A, Nichols TE, Jbabdi S, Westlye T, et al. Structural variability in the human brain reflects fine-grained functional architecture at the population level. Journal of Neuroscience. 2019; 39(31): 6136-6149.

4. Alexander-Bloch, Giedd JN, Bullmore E. Imaging structural co-variance between human brain regions. Nature Reviews Neuroscience. 2013; 14(5): 322-336.

5. Gupta L, Besseling MH, Overvliet GM, Hofman P, Louw Ad, Vaessen MJ, et al. Spatial heterogeneity analysis of brain activation in fMRI. NeuroImage: Clinical. 2014; 5: 266-276.

6. Finn ES, Shen X, Scheinost D, Rosenberg MD, Huang J, Chun MM, et al. Functional connectome fingerprinting: identifying individuals using patterns of brain connectivity. Nature neuroscience. 2015; 18(11): 1664-1671.

7. Wang K, Liang M, Wang L, Tian L, Zhang X, Li K, et al. Altered functional connectivity in early Alzheimer's disease: A resting-state fMRI study. Human brain mapping. 2007; 28(10): 967-978.

8. Arbabshirani MR, Kiehl K, Pearlson G, Calhoun VD. Classification of schizophrenia patients based on resting-state functional network connectivity. Frontiers in neuroscience. 2013; 7: 133. 
9. Cole DM, Smith M, Beckmann CF. Advances and pitfalls in the analysis and interpretation of restingstate FMRI data. Frontiers in systems neuroscience. 2010; 4: 8.

10. Crosson B, Ford A, McGreg KM, Cheshkov S, Li X, Walker-Batson D, et al. Functional Imaging and Related Techniques: An Introduction for Rehabilitation Researchers. Journal of rehabilitation research and development. 2010; 47(2).

11. Lee MH, Smyser CD, Shimony JS. Resting state fMRI: A review of methods and clinical applications. American Journal of neuroradiology. 2013; 34(10): 1866-1872.

12. Di X, Gohel S, Kim EH, Bisw BB. Task vs. rest-different network configurations between the coactivation and the resting-state brain networks. Frontiers in Human Neuroscience. 2013; 7: 493.

13. O'Connor E, Zeffiro TA. Why is Clinical fMRI in a Resting State? Frontiers in neurology. 2019; 10: 420.

14. Mueller S, Wang D, Fox MD, Yeo BTT, Sepulcre J, Sabuncu MR, et al. Individual Variability in Functional Connectivity Architecture of the Human Brain. Neuron. 2013; 77(3): 586--595.

15. Biswal B, Zerrin Yetkin F, Haughton VM, Hyde JS. Functionalconnectivityin the motor cortex of resting human brain using echo-planar MRI. Magnetic resonance in medicine. 1995; 34(4): 537-541.

16. Beckmann CF, DeLuca M, Devlin JT, Smith SM. Investigations into resting-state connectivity using independent component analysis. Philosophical Transactions of the Royal Society B: Biological Sciences. 2005; 360(1457): 1001-1013.

17. Calhoun VD, Adali T. Multisubject Independent Component Analysis of fMRI: A Decade of Intrinsic Networks, Default Mode, and Neurodiagnostic Discovery. IEEE reviews in biomedical engineering. 2012; 5: 60-73.

18. Power JD, Cohen AL, Nelson SM, Wig GS, Barnes KA, Church JA, et al. Functional network organization of the human brain. Neuron. 2011; 72(4): 665-678.

19. Lee MH, Hacker CD, Snyder AZ, Corbetta M, Zhang D, Leuthardt EC, et al. Clustering of resting state networks. PloS one. 2012; 7(7): e40370.

20. Damaraju E, Allen EA, Belger A, Ford JM, McEwen SJ, Mathalon D, et al. Dynamic functional connectivity analysis reveals transient states of dysconnectivity in schizophrenia. NeuroImage: Clinical. 2014; 5: 298-308.

21. Van Den Heuvel MP, Pol HEH. Exploring the brain network: A review on resting-state fMRI functional connectivity. European neuropsychopharmacology. 2010; 20(8): 519-534.

22. Roy AK, Shehzad Z, Margulies DS, Kelly AC, Uddin LQ, Gotimer K, et al. Functional connectivity of the human amygdala using resting state fMRI. Neuroimage. 2009; 45(2): 614-626.

23. Niu YW, Lin QH, Qiu Y, Kuang LD, Calhoun VD. Sample Augmentation for Classification of Schizophrenia Patients and Healthy Controls Using ICA of fMRI Data and Convolutional Neural Networks. In 2019 Tenth International Conference on Intelligent Control and Information Processing (ICICIP); 2019: IEEE. p. 297-302.

24. Qiu Y, Lin QH, Kuang LD, Zhao WD, Gong XF, Cong F, et al. Classification of schizophrenia patients and healthy controls using ICA of complex-valued fMRI data and convolutional neural networks. In International Symposium on Neural Networks; 2019: Springer. p. 540-547.

25. Hassanzadeh R, Calhoun D. Individualized Prediction of Brain Network Interactions using Deep Siamese Networks. In 2020 IEEE 20th International Conference on Bioinformatics and Bioengineering (BIBE); 2020: IEEE. p. 1065-1070.

26. Arbabshirani R, Plis S, Sui J, Calhoun D. Single subject prediction of brain disorders in neuroimaging: Promises and pitfalls. Neuroimage. 2017; 145: 137-165.

27. Chen G, Ward BD, Xie C, Li W, Wu Z, Jones JL, et al. Classification of Alzheimer Disease, Mild Cognitive Impairment, and Normal Cognitive Status with Large-Scale Network Analysis Based on Resting-State Functional MR Imaging. Radiology. 2011; 259(1): 213-221. 
28. Du W, Calhoun VD, Li H, Ma S, Eichele T, Kiehl KA, et al. High classification accuracy for schizophrenia with rest and task fMRI data. Frontiers in human neuroscience. 2012; 6: 145.

29. Tang Y, Wang L, Cao F, Tan L. Identify schizophrenia using resting-state functional connectivity: an exploratory research and analysis. Biomedical engineering online. 2012; 11(1): 50.

30. Iidaka T. Resting state functional magnetic resonance imaging and neural network classified autism and control. Cortex. 2015; 63: 55-67.

31. Khosla M, Jamison K, Kuceyeski A, Sabuncu MR. Ensemble learning with 3D convolutional neural networks for functional connectome-based prediction. Neuroimage. 2019; 199: 651-662.

32. Weis S, Patil KR, Hoffstaedter F, Nostro A, Yeo BT, Eickhoff SB. Sex classification by resting state brain connectivity. Cerebral cortex. 2020; 30(2): 824-835.

33. Zhang C, Dougherty CC, Baum SA, White T, Michael AM. Functional connectivity predicts gender: Evidence for gender differences in resting brain connectivity. Human brain mapping. 2018; 39(4): 1765-1776.

34. Pruett Jr JR, Kandala S, Hoertel S, Snyder AZ, Elison JT, Nishino T, et al. Accurate age classification of 6 and 12 month-old infants based on resting-state functional connectivity magnetic resonance imaging data. Developmental cognitive neuroscience. 2015; 12: 123-133.

35. Miranda-Dominguez O, Mills BD, Carpenter SD, Grant KA, Kroenke CD, Nigg JT, et al. Connectotyping: Model Based Fingerprinting of the Functional Connectome. PloS one. 2014; 9(11): e111048.

36. Button KS, Ioannidis JPA, Mokrysz C, Nosek BA, Flint J, Robinson ESJ, et al. Power failure: why small sample size undermines the reliability of neuroscience. Nature reviews neuroscience. 2013; 14(5): 365-376.

37. Ma S, Correa M, Li XL, Eichele T, Calhoun D, Adali T. Automatic Identification of Functional Clusters in fMRI Data Using Spatial Dependence. IEEE Transactions on Biomedical Engineering. 2011; 58(12): 3406-3417.

38. Liu, Liao X, Xia M, He Y. Chronnectome fingerprinting: Identifying individuals and predicting higher cognitive functions using dynamic brain connectivity patterns. Human brain mapping. 2018; 39(2): 902-915.

39. Kong R, Li J, Orban C, Sabuncu R, Liu H, Schaefer A, et al. Spatial topography of individual-specific cortical networks predicts human cognition, personality, and emotion. Cerebral cortex. 2019; 29(6): 2533-2551.

40. Bromley J, Guyon I, LeCun Y, Säckinger E, Shah R. Signature verification using a "siamese" time delay neural network. In Advances in neural information processing systems; 1994. p. 737-744.

41. Chung YA, Weng WH. Learning Deep Representations of Medical Images using Siamese CNNs with Application to Content-Based Image Retrieval. arXiv preprint arXiv:1711.08490\}. 2017.

42. Miller KL, Alfaro-Almagro F, Bangerter NK, Thomas DL, Yacoub E, Xu J, et al. Multimodal population brain imaging in the UK Biobank prospective epidemiological study. Nature neuroscience. 2016; 19(11): 1523-1536.

43. Jenkinson M, Bannister P, Brady M, Smith S. Improved optimization for the robust and accurate linear registration and motion correction of brain images. Neuroimage. 2002; 17(2): 825-841.

44. Andersson JLR, Skare S, Ashburner J. How to correct susceptibility distortions in spin-echo echoplanar images: application to diffusion tensor imaging. Neuroimage. 2003; 20(2): 870-888.

45. Salimi-Khorshidi G, Douaud G, Beckmann CF, Glasser MF, Griffanti L, Smith SM. Automatic denoising of functional MRI data: Combining independent component analysis and hierarchical fusion of classifiers. Neuroimage. 2014; 90: 449-468.

46. Beckmann CF, Smith SM. Probabilistic independent component analysis for functional magnetic resonance imaging. IEEE transactions on medical imaging. 2004; 23(2): 137-152. 
47. Griffanti L, Salimi-Khorshidi G, F. Beckmann C, Auerbach EJ, Douaud G, Sexton CE, et al. ICAbased artefact removal and accelerated fMRI acquisition for improved resting state network imaging. Neuroimage. 2014; 90: 449-468.

48. Du Y, Fu Z, Sui J, Gao S, Xing Y, Lin D, et al. NeuroMark: An automated and adaptive ICA based pipeline to identify reproducible fMRI markers of brain disorders. NeuroImage: Clinical. 2020; 28.

49. Brosch T, Tam R, Initiative ADN. Manifold Learning of Brain MRIs by Deep Learning. In International Conference on Medical Image Computing and Computer-Assisted Intervention; 2013; Berlin, Heidelberg: Springer. p. 633-640.

50. Krzywinski M, Schein J, Birol İ, Connors J, Gascoyne R, Horsman D, et al. Circos: an information aesthetic for comparative genomics. Genome research. 2009; 19(9): 1639-1645.

51. Wierenga M, Doucet E, Dima D, Agartz I, Aghajani M, Akudjedu N, et al. Greater male than female variability in regional brain structure across the lifespan. bioRxiv. 2020.

52. Forde J, Jeyachandra J, Joseph M, Jacobs R, Dickie E, Satterthwaite D, et al. Sex Differences in Variability of Brain Structure Across the Lifespan. Cerebral Cortex. 2020; 30(10): 5420-5430.

53. Save E, Poucet B. Hippocampal-parietal cortical interactions in spatial cognition. Hippocampus. 2000; 10(4): 491-499.

54. Jagaroo V. Mental rotation and the parietal question in functional neuroimaging: A discussion of two views. European Journal of Cognitive Psychology. 2004; 16(5): 717-728.

55. Koscik T, O’Leary D, Moser DJ, Andreasen NC, Nopoulos P. Sex differences in parietal lobe morphology: Relationship to mental rotation performance. Brain and cognition. 2009; 69(3): 451-459.

56. Adeli E, Zhao Q, Pfefferbaum A, Sullivan EV, Fei-Fei L, Niebles JC, et al. Representation Learning With Statistical Independence to Mitigate Bias. In Proceedings of the IEEE/CVF Winter Conference on Applications of Computer Vision; 2021. p. 2513-2523. 